\title{
Dark Matter: An Odd Need Created by Unsuitable Theories of Gravitation. The Higgs Quantum Space Dynamics Gravity Doesn't Need It
}

\author{
Jacob Schaf \\ Instituto de Física, Universidade Federal do Rio Grande do Sul (UFRGS), Porto Alegre, Brazil \\ Email: schaf@if.ufrgs.br
}

How to cite this paper: Schaf, J. (2018) Dark Matter: An Odd Need Created by Unsuitable Theories of Gravitation. The Higgs Quantum Space Dynamics Gravity Doesn't Need It. Journal of Modern Physics, 9, 1883-1905.

https://doi.org/10.4236/jmp.2018.910119

Received: July 11, 2018

Accepted: August 31, 2018

Published: September 3, 2018

Copyright $\odot 2018$ by author and Scientific Research Publishing Inc. This work is licensed under the Creative Commons Attribution International License (CC BY 4.0).

http://creativecommons.org/licenses/by/4.0/

\section{(c) (i) Open Access}

\begin{abstract}
The Higgs theory introduces the idea that space is filled up throughout by a quantum fluid medium, giving mass and mechanical properties to the elementary particles by the Higgs mechanism. This Higgs Quantum Space (HQS) thus governs the inertial motion of matter-energy and is locally their ultimate reference for rest and for motions. On the other hand, motion with respect to the local HQS and not relative motion is the origin of all the effects of velocity on matter, on light and on clocks. In previous works, the author has shown that the HQS, moving round the astronomical bodies according to a Keplerian velocity field $(G M / r)^{1 / 2} \boldsymbol{e}_{\phi}$, consistent with the local astronomical motions, accurately creates the observed gravitational dynamics and gives rise to all the observed effects of the gravitational fields on light and on clocks. The absence of the solar gravitational slowing on the GPS clocks and the absence of light anisotropy with respect to earth are both the signature of this HQS dynamics. In their orbital motion round the galactic center, the stars carry with them their Keplerian velocity fields. The present work shows that, due to the effects of this orbital velocity on the symmetry of the polarized star Keplerian velocity fields, the collective velocity field, created by them, does not fall with distance as the Keplerian profile $\left(r^{-1 / 2}\right)$. Depending on the distribution of the matter density, the velocity of the HQS and the stars can even increase with $r$. The non-Keplerian rotation of the galaxies thus is an intrinsic feature of the HQS dynamics gravitational mechanism, created without the need of dark matter.
\end{abstract}

\section{Keywords}

Dark Matter, Galactic Rotation, Gravitational Fields, Higgs Theory, 


\section{Introduction}

The structure of the galaxies is rather different from that of our solar system. While more than $99 \%$ of the mass of solar system is concentrated in the central (static) sun, more than $99 \%$ of the galactic mass is orbiting, within the thin galactic disk in the form of stars, around the galactic center, under the collective gravitational field created by these orbiting stars. In fact, the solar system too is disk shaped, however, the disk, formed by the planets, satellites and the asteroids all together contains less than $1 \%$ of the mass of the solar system. It however is sufficient to give rise to the Pioneer 10 and 11 anomalies as will be explained in Section 4.

Most galaxies are elliptic. Our Milky Way Galaxy is a large, old and barred spiral galaxy, with a central bulge extending out to about $10^{4}$ light years. The very center of this bulge wobbles a super-massive black hole with millions of solar masses. Beyond the bulge, a swarm of hundreds of billions of stars are all orbiting within a thin disk along direct circular equatorial orbits around the galactic center. The disk extends out to about 5/times $10^{4}$ light years, beyond which only several dwarf galaxies and globular star clusters are orbiting. The stars in the galactic disk are relatively isolated and well separated from each other by distances of several light years, so that the space within a galaxy is almost empty and the local star fields as well as the motion of the planets are only very weakly perturbed.

\section{The Non-Keplerian Rotation of the Galaxies Defies the Current Theories of Gravitation}

Many published works [1] [2] [3] display star orbital velocity profiles, as a function of the distance from the galactic center, for a number of galaxies. They all show that the orbital velocity of the stars in the galactic disk does not fall with distance from the galactic center as stipulated by the current theories of gravitation. In some galaxies, the orbital velocities even increases with the distance from the galactic center. Figure 1 displays the observed orbital velocity profile of the stars in the Milky-Way galaxy, together with a typical Keplerian rotation curve. The observed orbital velocity of the stars is much too fast to the gravitational pull and or the spacetime curvature, produced by the content and distribution of visible matter, to hold the Milky-Way together.

It is strange that the systematic discrepancy between the observed galactic rotation profiles and the theoretical predictions, instead of having raised suspicions about the current theories of space and gravitation, have lead to the odd idea that a huge amount of invisible dark matter, 5 times more than the total visible matter in a galaxy, must be responsible for this observed non-Keplerian 


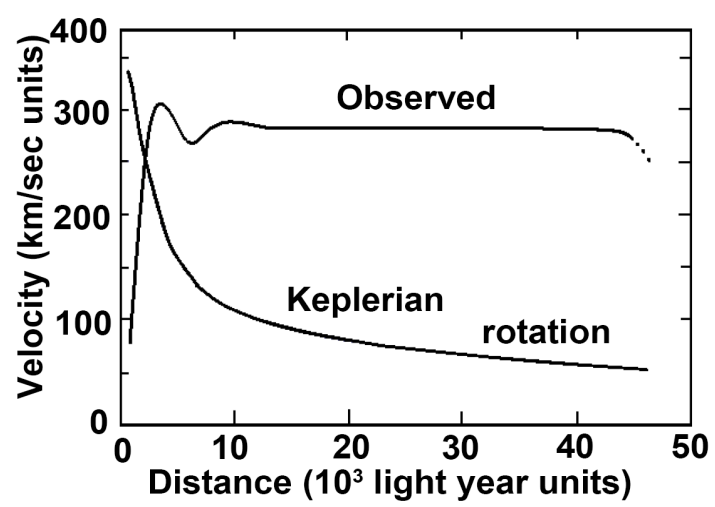

Figure 1. The observed orbital velocity profile of the Milky Way galaxy as a function of the distance from the galactic center, together with the typical Keplerian rotation curve, from Ref. [3].

rotation. The idea of the invisible dark matter is much too exotic. To present date nobody has idea what the nature of this dark matter could be. Some people think that it is formed by non-hadronic chargeless particles (WIMPS) that do not scatter, absorb or emit electromagnetic radiation. It is assumed that dark matter is constituted by particles with large masses and to interact with ordinary matter only by gravity. However, if dark matter interacts by gravity, why then is it not concentrated and found within stars and within galaxies? Some people even compute the distribution of dark matter in the form of halos round the galaxies that supposedly can cause the non-Keplerian galactic rotation. In Summary, the observed non-Keplerian rotation of the galaxies is actually among the most serious impasses in the current theories of space and gravitation [4] [5].

Another way to address the problem of the peculiar galactic rotation rate is by the gravitational potential. Several authors [6] have computed the gravitational potential $[U(r)]$ as a function of the radial distance from the galactic nucleus for our Milky Way galaxy as well as for other galaxies, with base in the current theories of space and gravitation, taking into account the observed matter distribution in these galaxies. Systematically all these computed gravitational potentials are inconsistent with the observed rotation rate of the galactic disk.

The profile of the gravitational potential can also be obtained by an empirical method, using the circular orbital velocity profile of the stars, like that depicted in Figure 1. According to the Virial theorem, the gravitational potential, of a spherically symmetric source, has a very simple relation with the circular orbital velocity $V_{o r b}(r)$. Although this approach is not exact, because galaxies are not spherically symmetric, it is sufficiently precise to provide some clear-cut conclusions. For a central inverse quadratic force field the relation between the gravitational potential and the circular orbital velocity is:

$$
U(r)=-V_{\text {orb }}^{2}(r)
$$

Figure 2 displays the gravitational potential $U(r)$, calculated, within the galactic border, with the help of Equation (1), using the observed velocity profile 


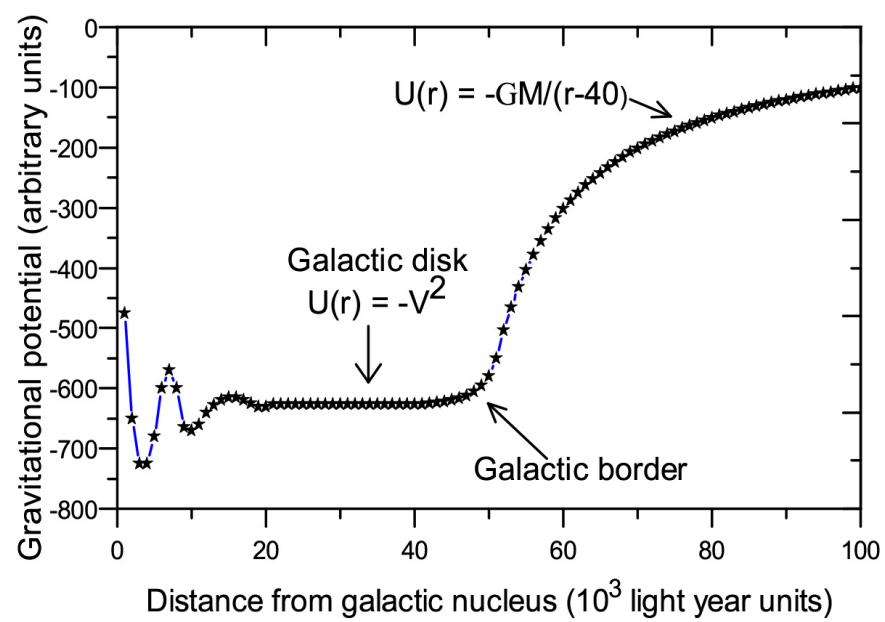

Figure 2. The figure depicts the gravitational potential for the Milky-Way galaxy, obtained by making use of Equation (1) and the observed orbital velocity profile in Figure 1. Within the galactic disk, this is to be seen as approximately the observed gravitational potential curve. However, beyond the galactic border, on from $(r-40) \times 10^{3}$ light years in Figure 2, the gravitational potential is assumed to follow nearly the usual $(1 / r)$ dependence, however only on from the galactic border.

of our Milky-Way galaxy in Figure 1. The galactic gravitational potential curve, obtained in this way, is to be seen as approximately the observed potential. Especial attention is called to the region within the galactic disk. The result shows that, in this region, the gravitational potential is closely leveled, which, from the Newtonian gravitation viewpoint, means that the force field toward the galactic center is almost zero $(\boldsymbol{F}(r)=m \boldsymbol{a}(r)=-\operatorname{grad} U(r) \sim 0)$. However, despite this absence, the stars are moving along circular orbits round the galactic center. From the usual mechanics point of view, this breaks fundamental principles of mechanics and seems absurd. Moreover, the spacetime curvature of GR too is well known to be unable to explain these observed orbital motions.

The impasse of the current theories with the observed non-Keplerian galactic gravitational dynamics can be solved in two ways: Or solid evidence must be found that enough dark matter is present at the right place or a fundamentally new gravitational theory must be found that explains this rotation without the need of dark matter. The perspective of success along the first possibility is hopelessly low. Therefore, the present work puts the hope on the second possibility. In the sixties of the past century a series of ground breaking scientific achievements, involving many brilliant scientists, have disclosed the origin of the inertial mass of the elementary particles [7] [8]. Peter Higgs has made the decisive step toward the solution. For this reason the theory is known by his name. The Higgs mechanism, providing inertial mass to the elementary particles, is of vital importance. Without it, the existence of our universe simply would be impossible. 


\section{Briefly the Higgs Quantum Space Dynamics Gravitational Mechanism}

The Higgs theory introduces the idea that space is filled up throughout by a quantum fluid medium, a quantum condensate of bosons, analogous to the superconducting condensate (SCC), here to be referred to by Higgs Quantum Space (HQS). Such quantum condensates are not ruled by classical hydrodynamics, however by the principles of quantum mechanics. Likewise the SCC, the HQS forms on spontaneously breaking the $U(1)$ gauge symmetry of the respective boson system and too can be described by a complex macroscopic Ginsburg-Landau like order parameter $\Phi=\phi \mathrm{e}^{i \theta}$. In terms of the Real and Imaginary components of $\Phi$ the characteristic form of the potential well, created by the condensate, has the form of a Mexican sombrero $V(\rho)=-n \rho+m \rho^{2}$, where $n$ is considerably larger than $m$ and $\rho=\Phi^{*} \Phi$. In this potential well, the minimum of the potential energy occurs not for $\rho=0$ however for $\rho=n / 2 m$. This remarkable feature is known as a non-zero vacuum expectation value. However, while the transition temperature of the SCC is very low and is stabilized by an energy gap of only e few meV, the Higgs quantum condensate or Higgs Quantum Space (HQS) is believed to have condensed shortly after the big-bang when the temperature fell through $10^{15} \mathrm{~K}$. It moreover is stabilized by a huge energy gap, which, according to the Glashow-Weinberg-Salam electroweak model, achieves more than $200 \mathrm{GeV}$.

The HQS couples to the vector bosons $W^{+}, W^{-}$and $Z_{0}$, confining and quantizing them, thereby giving them large rest masses by the Higgs mechanism. The HQS also couples indirectly to all the elementary particles porting hypercharges (quarks and leptons) by a Yukawa like mechanism, giving them mass. The Higgs mechanism is the perfect HQS analog of the Meissner effect [9] in superconductivity that couples to the electromagnetic (EM) field, confining and quantizing it and giving inertial mass to the EM field quanta (photons) within superconductors. The first clue that coupling of a field to a quantum condensate gives rise to mass terms was discovered by Anderson in superconductivity [10]. Gauge transformations of the superconducting order parameter $\Psi=\psi \mathrm{e}^{i \theta}$, in the presence of a magnetic field, reveal mass terms.

The Higgs theory introduces profound changes in Einstein's view about the nature of the empty space (vacuum). The HQS is much more than simply a local ultimate reference for rest and for motions. It literally governs the inertial motions of matter-energy. This essentially will say that velocity with respect to the local HQS and not relative velocity is the physical cause of all the effects of motion. The HQS materializes the local Lorentz frames, turning them into local proper Lorentz frames, intrinsically stationary with respect to the local HQS. On the other hand, references not stationary with respect to the local HQS are not proper LFs. The HQS also necessarily is responsible for the gravitational fields because it is mass that creates these fields. It is clear that the HQS plays a fundamental role in the microscopic physics of the quantum world as well as in 
the macroscopic world of gravitation. The HQS thus is the link that units quantum physics and gravitation.

Einstein's model of the free-falling inertial references (IRs), proposed in General Relativity to explain the origin of the gravitational dynamics has a serious shortcoming. It cannot give rise to the observed gravitational pull. It cannot because the local free-fall velocity from the infinite $v_{f f}=(2 G M / r)^{1 / 2}$ (local escape velocity) at any point $r_{0}$ within the gravitational field is constant $\left(\mathrm{d} v_{f f} / \mathrm{d} t \mid r_{0}=0\right)$. Therefore, a body brought to rest at any given position in the gravitational field and then released, will remain in equilibrium there. Any perturbation however will trigger a runaway departure upward or downward. In fact, within a gravitational field any matter body that is not thrust by an external force, must be considered as a free-body. This proves that the nature of the real constant upward force, necessary to prevent the free-fall of such a free body and acting without producing any upward velocity along its instantaneous direction, is clearly of a centripetal nature. In its turn, the downward reaction force, known as gravitational pull, necessarily is of a centrifugal nature. This will say that the local inertial references are not free falling; however are rotating round an over-head axis. The gravitational pull is a spherically symmetric inward centrifuge effect.

In previous publications [11] [12] [13] [14] [15], the author shows that the absence of the gravitational slowing of the GPS clocks by the solar field as well as the absence of light anisotropy with respect to earth demonstrate that the HQS is moving round the sun according to a Keplerian velocity field, consistent with the planetary motions, in which the value of the local velocity is spherically symmetric. This Keplerian velocity field of the HQS is shown to accurately produce all the effects of the gravitational fields on matter, on light and on clocks. In terms of the usual spherical coordinates $[r, \theta, \phi]$ the velocity field of a spherically symmetric source has the very simple form:

$$
\boldsymbol{V}(r)=(G M / r)^{1 / 2} \boldsymbol{e}_{\phi}
$$

This Keplerian velocity field is the quintessence of the gravitational fields.

In this Keplerian velocity field, the velocity of the HQS increases for decreasing radial coordinate $r$ at all positions $[r, \theta, \phi]$ round the gravitational source from the equator to the Poles. Therefore, the local distribution of velocity (velocity gradient) through any infinitesimal region in any $[r, \theta]$ plane, which is parallel to the wave fronts of a particle moving along the $\phi$ coordinate, is consistent with rotation of the local HQS and necessarily of the local inertial reference (IR) round an overhead axis. The angular velocity round this overhead axis points along $+\theta$ and can be shown (Refs. $(11,12)$ to be located in the ordinary space at a distance $r$ above the position of the particle. The local rotating references are inertial references because it is the HQS, ruling the inertial motion of matter that is itself so rotating. This will say that a free particle, initially stationary in the ordinary space within this Keplerian velocity field (earth-based laboratory) is implicitly moving with respect to the local (moving) 
HQS at a velocity $\left(\boldsymbol{V}=-(G M / r)^{1 / 2} \boldsymbol{e}_{\phi}\right)$. This velocity is implicit because it cannot bve described with respect to the ordinary space. The implicit velocity vector will rotate with the local IR round the same overhead axis at an angular velocity $\left(\boldsymbol{W}(r)=\left(G M / r^{3}\right)^{1 / 2} \boldsymbol{e}_{\theta}\right)$. Consequently, the particle will develop an increasing velocity component along $r$, the acceleration being given by $\boldsymbol{W}(r) \times \boldsymbol{V}(r)=-\left(G M / r^{2}\right) \boldsymbol{e}_{r}$. This is exactly the expression for the centrifugal acceleration in a rotating (non-inertial) reference. Here, the non-inertial reference (laboratory) is implicitly rotating (oppositely) with respect to the local IR that is truly rotating in the ordinary space. Note that this centrifugal acceleration points, from every point, toward the gravitational center. On the other hand, a particle of mass $m$, stationary with respect to the ordinary space within the Keplerian velocity field Equation (2), will implicitly be moving along a circular path, within the local truly rotating IR, under a real upward centripetal force $\boldsymbol{F}_{c p}(r)=+\left(m G M / r^{2}\right) \boldsymbol{e}_{r}$. The downward reaction force to this real upward centripetal force is a fictitious force, a genuine centrifugal force (gravitational pull) $\boldsymbol{F}_{c f}=-m \boldsymbol{g} \boldsymbol{e}_{r}$. Please see Refs. [11] [12] [13] for the full details. In this HQS dynamics scenario the gravitational pull is identically a usual centrifugal pull toward the gravitational center.

The Keplerian velocity field of the HQS Equation (2), governing the inertial motion of matter-energy, is among all imaginable physical mechanisms the only one that is capable of generate and implement the outside-inside and inside-outside centrifuge scenario, observed within the gravitational fields. No other theory of gravitation can give rise to this intriguing inertial dynamics that is so difficult to grasp. The non-uniform velocity of the HQS in the Keplerian velocity field is the key feature that implements this apparently magic physics. In conclusion, the gravitational pull is nothing than simply and exactly a centrifugal pull, generated by the Keplerian velocity field of the HQS. In view of these facts, the present work associates together the central idea of GR, according to which the gravitational pull is an inertial pull and the central idea of the Higgs theory, according to which the HQS governs the inertial motion of matter-energy and replaces Einstein's spacetime curvature by a Keplerian velocity field of the HQS.

In the solar Keplerian velocity field, the orbiting earth, as well as the other planets of the solar system are very closely stationary with respect to the local moving HQS (and the local proper Lorentz frames). This predicts the absence of the gravitational slowing of the GPS clocks by the solar field, as observed. It also predicts the well-known absence of light anisotropy with respect to earth and all the other observed effects of the gravitational fields on light and on clocks. Current theories explain the absence of the solar gravitational slowing on the GPS clocks in terms of the principle of equivalence. However, the GPS clocks too are moving round earth in exactly the same conditions as round the sun, which too should cancel the gravitational slowing by the earth field. This cancelation however is not observed. The GPS clocks move round earth in orbits that make 55 degrees with the earth's equator. This is the relevant difference that is responsible for the non-cancelation of the gravitational slowing of the GPS 
clocks by the earth's field. Please see Refs. [11] [12] [13] for the detailed explanation. The absence of the solar gravitational slowing of the GPS clocks and the absence of light anisotropy with respect to the planet earth are both authentic signatures of the true physical mechanism of gravity in action.

The velocity field of the HQS of a galaxy results from the composition of a huge number of the stellar Keplerian velocity fields Equation (2). In order to these stellar velocity fields to build up the galactic velocity field, the individual star velocity fields must be fairly well polarized. They must all be rotating in the same sense as the galactic rotation, round axes that are dominantly parallel to that of the galaxy. In the solar system, the planetary Keplerian velocity fields of the HQS are polarized to a high degree. This is evident from the fact that the planets move all in the same sense along circular orbits round the sun, within the plane of the solar system. Moreover, the planets rotate round nearly parallel axes and the planetary satellites move all in the same sense round these axes along nearly circular orbits closely within the plane of the solar system. The stars in the galaxies too orbit all in the same sense round the galactic center along closely circular orbits within the galactic plane. It seems reasonable to assume that the stellar Keplerian velocity fields rotate all in the same sense as the galactic rotation, round axes dominantly parallel to the galactic rotation axis.

The coming Sections will show that, due to the orbital velocity, the collective galactic velocity field of the HQS, created by the orbiting stars, has not the Keplerian form. It does not fall with the radial distance $r$ as in the Keplerian velocity field $\left(r^{-1 / 2}\right)$. The reason is that, from the view of an external observer, the orbital velocity $v_{\text {orb }}$ of each star subtracts from its local symmetric Keplerian velocity field Equation (2) toward the inner side of the orbit, however adds up to it toward the outer side. Because of this asymmetric effect, the velocity profile in the galactic disk of most galaxies becomes nearly constant with $r$ and, in some galaxies, it even increases with the radial distance. It also naturally depresses the velocity profile within a central region as observed in all galactic velocity profiles. It can even generate a central region with retrograde rotation, as observed in many galaxies. The next Section 3 makes a quantitative analysis for the case of a binary star system that reveals a key feature, which is essential to understand the non-Keplerian rotation of the galaxies. Section 4 constructs a qualitative model that reproduces the observed non-Keplerian rotation of the galaxies without the need of dark matter.

\section{Binary Stars Contain a Key Feature that Unveils the Origin of the Non-Keplerian Rotation of the Galaxies}

The crucial question to be answered here is: Why do the orbital velocities of the planets decrease with distance from the gravitational center according to $(1 / r)^{1 / 2}$, while the orbital velocities of the stars in the galactic disk fall less than

$(1 / r)^{1 / 2}$ and in many galaxies do not fall at all or even increase with distance $r$ ? This is the observational fact that the current theories of gravitation cannot 
explain without postulating a huge amount of dark matter. Here the goal is showing that the gravitational theory, based in the HQS dynamics gravitational mechanism, predicts this non-Keplerian galactic gravitational dynamics without the need of dark matter. It even reveals incredible details. The origin of this behavior is related with the fact that motion of a gravitational source gives rise to important effects on the velocity field of the HQS, creating the gravitational fields, even for relatively low velocities. In the current theories only very high velocities, comparable to the velocity of light, cause significant changes.

In order to highlight the relevance of the effects of motion of the gravitational sources on their HQS velocity field in a system of bodies gravitating in their self-consistent velocity field, let us begin with the simple case of a binary system of two equal stars. Assume that the velocity fields of both stars are perfectly polarized (velocity fields Equation (2) rotating in the same sense round parallel axes) and that the stars are both moving along the same circular equatorial orbit round the center of mass (CM). Please see a sketch in Figure 3. These stars both will be moving along a same circular orbit, together with the local HQS in the collective velocity field of the HQS, created by them. They are each one stationary with respect to the local HQS. The gravitational dynamics of such a binary system has the virtue of disclosing very clearly the effects of the orbital motion of the sources on their velocity fields within the collective gravitational field of the binary system, which is seen by an observer in the non-rotating (XY) reference. These effects unveil the essential clue that is the key to understand the origin of the non-Keplerian galactic gravitational dynamics.

Consider two equal stars with equal masses $M_{1}=M_{2}=M$, moving in the same circular orbit round their center of mass (CM) and the velocity fields, creating their individual gravitational fields, rotating in the same sense as the orbital motion round parallel axes attached to the center of these gravitational sources, as shown in Figure 3. These stars will be com-moving with the local HQS along circular orbits round the CM in the equatorial plane of the collective velocity field of the HQS, creating the gravitational field of the binary. Note that this configuration (axes forming less than 90 degrees) of the velocity fields is the only one leading to a stable bound system. It can be shown that stars with oppositely (anti-parallel) rotating velocity fields of the HQS cannot form a stable bound system, because, on approximating, their opposite velocity fields would add up and gradually cancel, thereby canceling their negative gravitational potentials. Such systems in fact must be anti-gravitating.

The gravitational dynamics of the binary system can be well described by Newtonian mechanics. Balance of the mutual Newtonian gravitational forces $G M^{2} /\left(2 x_{0}\right)^{2}$ on each star and of the opposite centripetal forces $M v_{o r b}^{2} / x_{0}$ leads to the observed orbital velocity $v_{\text {orb }}$ of each star round the CM:

$$
v_{\text {orb }}=\frac{1}{\sqrt{2}}\left(G M / 2 x_{0}\right)^{1 / 2}
$$

where $2 x_{0}$ is the distance between the two stars and where $x_{0}$ is a positively defined length (please see Figure 3), which is the distance to the CM. Consider 


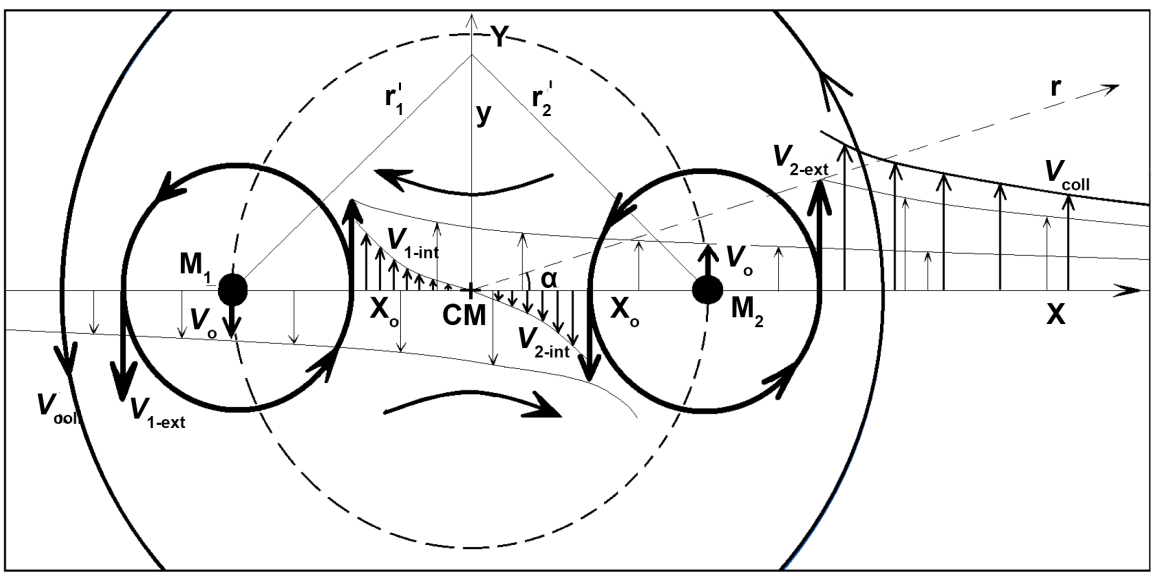

Figure 3. View of the velocity fields of the HQS in the equatorial plane of two masses $M_{1}=M_{2}=M$, moving in the same sense along the same circular orbit round the center of mass (CM) within the equatorial plane of the combined velocity field. The arrows, indicating the velocities are plotted all to scale. Note that, from the perspective of the observer in the non-rotating [X, Y] coordinate axes, the velocity of the HQS, due to the velocity fields of $M_{1}$ and or of $M_{2}$, are larger outside than inside the binary orbit.

now a small test particle $m$ moving round an equal, however isolated and static mass $M(M \gg m)$ in a circular equatorial orbit having the same radial distance $2 x_{0}$ between $m$ and $M$ as between the masses in the binary. The orbital velocity $v_{o r b}^{\prime}$ of this test particle must be:

$$
v_{\text {orb }}^{\prime}=\left(G M / 2 x_{0}\right)^{1 / 2}=\sqrt{2} v_{\text {orb }}
$$

It immediately is seen that the velocity $v_{o r b}^{\prime}$ in Equation (4) is considerably larger than $v_{o r b}$ in Equation (3).

From the viewpoint of the Newtonian gravitational theory there is obviously nothing wrong with Equations (3) and (4). On the other hand, from the viewpoint of the HQS-dynamic gravitational mechanism, the central gravitational source in Equation (4) practically remains stationary at the CM. Therefore this Equation gives the local velocity of the HQS in the Keplerian velocity field round a static mass $M$, exactly as stipulated by Equation (2). However, in the dynamic case of the binary stars, Equation (3) too gives the value of the velocity of the HQS, at the site of $M_{1}$ and at the site of $M_{2}$, due to the respective companion star. Otherwise, it would be impossible to their orbits to be circular. Hence, both Equations (3) and (4) describe the velocity of the HQS at the orbits in the respective velocity fields. However, why are these velocities so different?

In the view of the present work, the difference between Equation $s$ (3) and (4) unveils a key feature that is responsible for the non-Keplerian rotation of the galaxies. This difference discloses the effect of the relatively slow orbital velocity of the gravitational sources on the velocity field of the HQS, creating their respective gravitational fields as well as on the collective velocity field $V_{\text {coll }}$, responsible for the gravitational dynamics of the binary as viewed by an observer in the static non-rotating (XY) reference (please see Figure 3). 
However, in the opinion of an observer, moving together with one or the other source of the binary, the respective sources are stationary with respect to the local HQS and the velocity field of the HQS, creating the local gravitational field of each individual source, is closely spherically symmetric, as given by Equation (2), excepting only for small tides. This observer can confirm these facts by light anisotropy experiments and by the rate of his atomic clocks. Nevertheless, from the viewpoint of an observer stationary in the static non-rotating (XY) coordinate axes (please see Figure 3), which constitute the reference with respect to which the collective velocity field of the binary must be described, the velocity fields of the individual stars are considerably asymmetric. The value of the velocity of the HQS, at points on the $\mathrm{X}$ axis, is much larger outside the orbit of the binary than inside. Moreover, the sense of the velocity of the HQS inside the orbit is opposite to the orbital motion as well as opposite to the considerably higher velocity outside the binary. The circumstance is analogous to the observations of the wheels of a moving car in the view of a resting street observer and of the passenger in the car. In the view of the street observer the lower part of each wheel is stationary on the street, while the upper part is moving at a velocity two times the velocity of the car. However, in the view of the passenger, inside the car, the wheels are rotating symmetrically round their axes.

All these features are fundamental to understand the non-Keplerian gravitational dynamics of galaxies. In the case of the binary, the only possible reason for the reduced velocity of the HQS, at the position of $M_{1}$ or of $M_{2}$ is the orbital velocity of the companion star round the center of mass (CM). In fact the orbital velocity of the sources, given by Equation (3), subtracts from the spherically symmetric velocity fields Equation (2) in the reference of each source inside the orbit and adds up to it outside the orbit, as depicted in Figure 3.

In order to put in evidence the full effects of motion of the gravitational sources on their velocity fields, consider now, in addition, a small test particle, moving in the collective velocity field of the binary along a direct circular orbit within the orbital plane of the binary. If this orbital motion takes place sufficiently far away from the binary, the asymmetries of the binary field will be minimized. The orbital velocity $v$ of such a test particle is of course given by:

$$
v(r)=\sqrt{\frac{G 2 M}{r}}
$$

where $r$ is the distance on from the CM of the binary. For large $r$ Equation (5) too corresponds well to the velocity of the HQS round the binary, however in the collective velocity field round the binary:

$$
V_{\text {coll }}(r)=\sqrt{\frac{G 2 M}{r}}
$$

In order to reconcile the addition of the velocity fields of $M_{1}$ and $M_{2}$ outside the binary with the velocity in the collective velocity field, given by Equation (6), the same orbital velocity that reduces the velocity fields toward the inner side, must increase it outward the binary orbit. It must increase the velocity field $V_{1}$ 
toward the left-hand side of $M_{1}$ and also increase the velocity field $V_{2}$ toward the right-hand side of $M_{2}$ as plotted in Figure 3. At distances $r$ much larger than $2 x_{0}$, the addition of the velocity fields of $M_{1}$ and $M_{2}$ must reproduce the value, given by Equation (6). Note however that the addition of the velocity fields, due to different sources $M_{1}$ and $M_{2}$ along the axis of $X$, must satisfy the sum rule:

$$
V(r)=\sqrt{\left|V_{1}^{2}\left(r_{1}\right) \pm V_{2}^{2}\left(r_{2}\right)\right|}
$$

The reason of this sum-rule is the fact that the velocity field of the HQS depends on the square-root of the source mass and distances. Taking into account the sum rule Equation (7), the addition of the velocity fields of $M_{1}$ and $M_{2}$, at the $+X$ axis and for large $x$ (large $r$ in Figure 3), takes the form:

$$
V_{\text {coll }}^{2} \sim \frac{G 2 M}{r}=\frac{1}{2} \frac{G M_{1}}{x_{0}+x}+V_{\text {ext }, 2}^{2}
$$

Considering that, along the axis of $X, r=x$ and the distance from $M_{2}$ is $x-x_{0}$ and solving for $V_{e x t, 2}$ for points on the $+X$ axis, for $x \gg x_{0}$, the velocity field, produced by $M_{2}$ is approximately given by:

$$
V_{e x t, 2} \sim \sqrt{\frac{3}{2}} \sqrt{\frac{G M}{x-x_{0}}}
$$

A totally similar result is found toward the left hand side of $M_{1}$ and hence $\left|V_{\text {ext }, 1}\right|=\left|V_{\text {ext }, 2}\right|=V_{\text {ext }}$.

Inserting the result of Equation (9) into Equation (8) and adding according to Equation (7), the velocity field of the binary to the right hand side of $M_{2}$ (along points on $+X$ ), for large $X$, which means large $r$, is:

$$
V_{\text {coll }}=\sqrt{\frac{3}{2} \frac{G M}{x-x_{0}}+\frac{1}{2} \frac{G M}{x_{0}+x}} \sim(G 2 M / r)^{1 / 2}
$$

This reproduces the result of Equation (6) for large $r$. The procedure to obtain $V_{\text {coll }}$ to the left hand side of $M_{1}$ is analogous and the result is identical.

This result shows that effectively the same orbital velocity that, in the view of the static observer in the (XY) reference, reduces the velocity fields of $M_{1}$ and or $M_{2}$ (Equation (2)) by a factor $(1 / 2)^{1 / 2}$ toward the inner side in Figure 3, enhances the velocity fields of $M_{1}$ and $M_{2}$ by a factor $(3 / 2)^{1 / 2}$ toward the outer side. This is of course not for nothing. Clearly, the reason is the orbital velocity of the sources. This result shows that, while the contribution of $M_{1}$ to the collective velocity field, at points on the axis of $X$ toward the right hand side of $M_{2}$ is only (1/4) $V_{\text {coll }}$, the contribution of $M_{2}$ is $3 / 4 V_{\text {coll }}$. An analogous conclusion is valid at the left hand side of $M_{1}$, however with the roles of $M_{1}$ and $M_{2}$ interchanged. Hence, while, from the viewpoint of the static (XY) reference, the effects of the orbital velocity on the velocity fields of the individual stars change considerably the symmetry of the velocity fields of the individual stars, they do not affect significantly the value of the collective (total) velocity field far outside from the binary. The effective collective velocity field, outside de binary, is closely the same as in the case, in which the two sources are static. 
In conclusion, toward the inner side of the binary, on the axis of $X$, the orbital velocity $v_{o r b}$ subtracts from the spherically symmetric velocity fields (Equation (2)) of each source, in consonance with Equation (7):

$$
V_{\text {int }}=\sqrt{\frac{G M}{x^{\prime}}-v_{\text {orb }}^{2}}=\frac{1}{\sqrt{2}} \sqrt{\frac{G M}{x^{\prime}}}
$$

where $x^{\prime}$ is the distance on from either $M_{1}$ or $M_{2}$ toward the inner side and beyond respectively toward $+\infty$ or $-\infty$ along the $X$ axis. However, outward the binary, either toward the left on from $M_{1}$ in Figure 3 or toward the right on from $M_{2}$, the orbital velocity adds up to the respective spherically symmetric velocity field Equation (2), in consonance with Equation (7):

$$
V_{\text {ext }}=\sqrt{\frac{G M}{x^{\prime}}+v_{o r b}^{2}}=\sqrt{\frac{3}{2}} \sqrt{\frac{G M}{x^{\prime}}}
$$

where $x^{\prime}$ is the distance toward the left on from either $M_{1}$ or toward the right on from $M_{2}$ in Figure 3, along the axis of $X$ and to respectively $-\infty$ or $+\infty$.

In the view of an observer, stationary on the $X$ axis of the non-rotating (XY) reference, far away at the right hand side of $M_{2}, M_{2}$ contributes (3/2)GM/ $x^{\prime}$ to the total gravitational potential of the rotating binary, the total value of which is $U\left(x^{\prime}\right)=G 2 M / r$, while $M_{1}$ contributes only $(1 / 2) G M /\left(2 x_{0}+x^{\prime}\right)$ to it. An analogous conclusion is valid at the left hand side of $M_{1}$, where however the roles of $M_{1}$ and $M_{2}$ are interchanged. The origin of the difference between the contributions of $M_{1}$ and $M_{2}$ to the effective gravitational potential is, besides their different positions, principally their orbital velocities. This view of the static (XY) observer is of fundamental importance, because it is exactly the view of the gravitational dynamics we usually make about the stars within galaxies. Note that in the view of the current gravitational theories, the effect of the low orbital velocities on the gravitational potential of the individual sources is totally irrelevant. In these theories the gravitational potential depends only on the position and not on the velocity of the sources. However, the total gravitational potential far away outside the binary, predicted by both approaches, are the same $U \sim G 2 M / r$.

The results expressed by Equations (11) and (12) and plotted in Figure 3 are a unique feature of the present HQS-dynamic gravitational mechanism. It arises from the fact that the gravitational dynamics is created by the Keplerian velocity fields of the moving sources. None of the current theories of gravitation can give rise to the features expressed by Equations (11) and (12). These equations show that, while the velocity field, created by $M_{2}$ at its left hand side, is downward and has the value $-(1 / 2)^{1 / 2}\left(G M / x^{\prime}\right)^{1 / 2}$, at the right hand side it is upward and has the value $+(3 / 2)^{1 / 2}\left(G M / x^{\prime}\right)^{1 / 2}$. In these expressions $x^{\prime}$ is the distance on from the position of $M_{2}$ to either sides. Hence, going in Figure 3 along the positive $X$ axis, on from the $\mathrm{CM}$, there is an upward velocity step $\Delta V$, from $-(1 / 2)^{1 / 2}\left(G M / x^{\prime}\right)^{1 / 2}$ to $+(3 / 2)^{1 / 2}\left(G M / x^{\prime}\right)^{1 / 2}$, created by $M_{2}$ : 


$$
\Delta V=\left[\sqrt{\frac{3}{2}}+\sqrt{\frac{1}{2}}\right] \sqrt{\frac{G M}{x^{\prime}}} \sim 1.93 \sqrt{\frac{G M}{x^{\prime}}}
$$

Note that this addition is not ruled by Equation (7) because the velocities are of the same mass. Going along the $X$ axis toward the left hand side in Figure 3, on from the CM, there is an analogous velocity step at the position of $M_{1}$, however increasing the (opposite) downward velocity (please see Figure 3). Note also that the magnitude of the velocity step depends on the square-root of the mass $M$ as well as of $x^{\prime}$, the distance from this mass. For instance, the velocity of the HQS in the solar Keplerian velocity field achieves $436 \mathrm{~km} / \mathrm{sec}$ at the solar surface. However, at distances of the first neighbor star (Alfa Centaurus) the velocity is only about 60 meters/sec. The sun thus creates on its surface a velocity step of $872 \mathrm{~km} / \mathrm{sec}$ from side to side and only of about 120 meters/sec at the distances of Alfa Centaurus.

The velocity steps created by the stars in the binary are a key feature that is fundamental in the building up of the velocity field of the HQS, ruling the non-Keplerian galactic gravitational dynamics. In this building up, it is important to perceive that the upward orbital velocity step of $M_{2}$ in Figure 3 and given by Equation (13), does not only increase the upward velocity at the right hand side of $M_{2}$, according to Equation (12), however also decreases the downward velocity at its left hand side, as given by Equations (11). This shows that the total magnitude of the velocity step, created by $M_{2}$, is the same as in the static situation, however shifted by the orbital velocity.

The obvious conclusion from the above analysis is that the Keplerian velocity fields of the individual stars in the galactic disk act all in the sense of opposing the Keplerian reduction $(1 / r)^{1 / 2}$ of the galactic velocity field. In this modified Keplerian dependence, the velocity outside the orbit that is increased by the orbital velocity, as well as the velocity inside the orbit, decreased by the orbital velocity, both act together in the sense of locally opposing the Keplerian decrease of the velocity as a function of the distance from the galactic center. In some galaxies this effect is strong enough to invert the velocity gradient so much that the velocity, instead of decreasing, effectively increases with $r$. However, beyond the border of the galactic disk, where the mass density falls strongly toward zero, the galactic velocity field of course tends to recover the Keplerian $(1 / r)^{1 / 2}$ dependence.

In-between the orbiting stars of the binary system the velocity fields of the HQS, due to $M_{1}$ or $M_{2}$ at the axis of $X$, are opposite to each other (please see Figure 3). Moreover, they are reduced by their orbital velocity given by Equation (3), as given by Equation (11). Along the $X$ axis, the addition of these internal velocity fields, according to Equation (7), shows that, close to $M_{1}$, the upward velocity field of $M_{1}$ dominates and close to $M_{2}$ the downward velocity field of $M_{2}$ is dominant. The internal velocity field rotates in the opposite sense to that of the external velocity field. In-between the two masses, the resultant collective velocity field, as a function of the position $x$ and along the axis of $X$, is 
given by:

$$
V_{\text {int }}(x)=\sqrt{\left|V_{1}^{2}-V_{2}^{2}\right|}=\sqrt{\left|\frac{1}{2} \frac{G M_{1}}{x_{0}+x}-\frac{1}{2} \frac{G M_{2}}{x_{0}-x}\right|}
$$

where $x_{0}$ is positively defined, but $x$, in the term of $M_{1}$, is initially negative, but becomes positive beyond the origin (CM), while, in the term of $M_{2}, x$ is initially positive, but becomes negative below the origin of the (XY) system. If $M_{1}=M_{2}$, the resultant velocity at the origin is zero $\left(V_{\text {int }}(x=0)=0\right)$. If $x=-x_{0}, V_{\text {int }}(x)$ points upwards and is large near $M_{1}$ (see Figure 3) and for $x=+x_{0}, V_{\text {int }}(x)$ points downwards and is large near $M_{2}$. The opposite velocity field within the binary orbit is not at all strange. In many galaxies, a central region rotates oppositely to the external disk. NGC 7331 is a well-known example in which the central bulge is rotating oppositely to the galactic disk. [16]

In the other regions of the XY plane, the velocity field is strongly affected by continuity and conservation of volume. However, for large $r$ the velocity field tends to the form given by Equation (6). Along the axis of Y, the velocity of the HQS increases continuously for decreasing $y$, however the velocity gradient gradually falls and vanishes at $y=0$. The difficulty to describe the velocity field in such regions however is specific to the binary system. For more symmetric systems, like galaxies, this problem becomes irrelevant.

Finally, the collective velocity field of the HQS, generated by a binary system, necessarily is locally non-symmetric about the center of mass. On rotating in the ordinary space, binaries naturally induce oscillations ( $4 \pi$ per orbit) in the magnitude of the collective velocity field of the HQS in the (XY) plane. These oscillations of the velocity field and of the gravitational field intensity expand at the velocity of light and are known as gravitational waves. Hence, while the rotation of circularly symmetric systems does not create oscillations of the velocity field and no gravitational waves, binary systems naturally create oscillations and emit gravitational waves. They are the most efficient mechanism in nature generating gravitational waves.

\section{A Model for the Observed Non-Keplerian Rotation of the Galaxies, without the Need of Dark Matter}

In the solar system, the sun and the planets rotate all in the same sense round axes closely parallel to the axis of the solar system. Moreover, the orbital motions of the planets round the sun and the orbital motions of their satellites all move along direct and closely circular equatorial orbits within the plane of the solar system. These orbital motions demonstrate that the Keplerian velocity fields, creating the gravitational fields of the sun, of the planets as well as of their satellites are highly polarized. The only significant deviation from this highly ordered dynamics is the rotation of Uranus and the orbital motions of its satellites. This however visibly is an exception, visibly due to a strong perturbation, like a catastrophic collision. In order to build up the galactic velocity field of the HQS, the velocity fields Equation (2) of the individual stars, too must be fairly 
well polarized. It is well-known that the stars in the galactic disk too are moving all along nearly direct circular orbits in the same sense round the galactic center. Presently the orbits of their planets are largely unknown. It however seems quite reasonable to assume that the rotation axes of the stellar velocity fields of the HQS, creating the stellar gravitational fields, are fairly well polarized. Their velocity fields of the HQS must be rotating dominantly in the same sense as the rotation of the galaxy, round axes that make an angle lower than 90 degrees with the rotation axis of the galaxy. The rotation axis of our solar system is well-known to make about 63 degrees with respect to the rotation axis of the Milky-Way galaxy. This angular deviation may be related with the spiraled structure of our galaxy. Adequate addition of the star velocity fields must generate the galactic velocity field of the HQS that rules the galactic gravitational dynamics.

The previous Section 3 makes a quantitative analysis of the gravitational dynamics of a binary star system from the viewpoint of the HQS-dynamics gravitational mechanism. Now, consider an increasing number of stars, moving in the same sense along the same circular orbit round the CM and their velocity fields of the HQS rotating all in the same sense as their orbital motions, round axes that are all parallel to each other. Figure 4 displays a sketch of the collective velocity field, created by a system of eight equal stars. The figure shows that, with an increasing number of stars, the velocity in the collective velocity field increases and becomes smoother.

Note that the velocity in the internal collective velocity field is opposite to the velocity in the external collective velocity field. A stagnation point exists at the center as well as between each pair of stars. Each one of the eight stars is stationary with respect to the local moving HQS in the collective velocity field. These eight stars simply are carried round the CM by the HQS in the local collective velocity field.

In order to extend the model of the star-loop in Figure 4 closer to the realistic situation of the galactic disk, consider now multiple concentric and coplanar circular orbit loops with larger and larger radii and each loop containing a very large number of stars, rotating all in the same sense and moving in the same sense round the CM. Figure 5 is a representation of the velocity profile of the velocity field of the HQS as well as of the estimated effective velocity of the stars (blue line) through four successive loops in an intermediate region of $r$ for a case in which the density of stars is constant with the distance $r$ from the galactic center. This means equal linear density of matter along each star loop and equal velocity steps at each loop.

Precise computation of the velocity field of a galaxy, containing hundreds of billions of stars, obviously requires formidable computational means. Here only a qualitative estimate is possible, which however convincingly shows that the HQS-dynamics gravitational mechanism consistently and naturally leads to the observed non-Keplerian rotation of the galaxies and moreover reveals some 


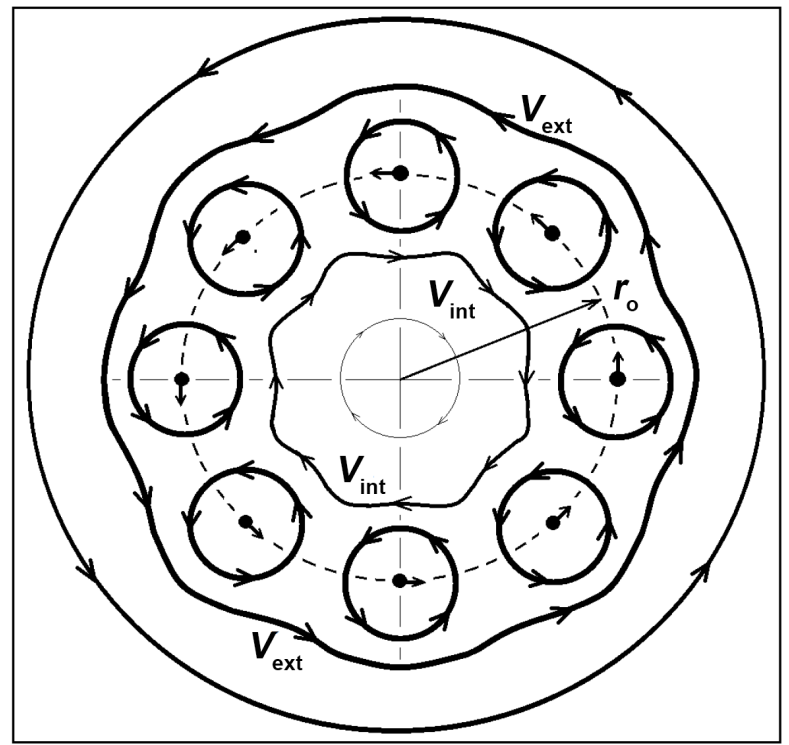

Figure 4. Sketch of the collective velocity field, generated by the polarized velocity fields of eight equal stars, moving together with the local HQS along the same circular orbit round the CM. The strength of the velocity field is qualitatively indicated by the wideness of the velocity tracks.

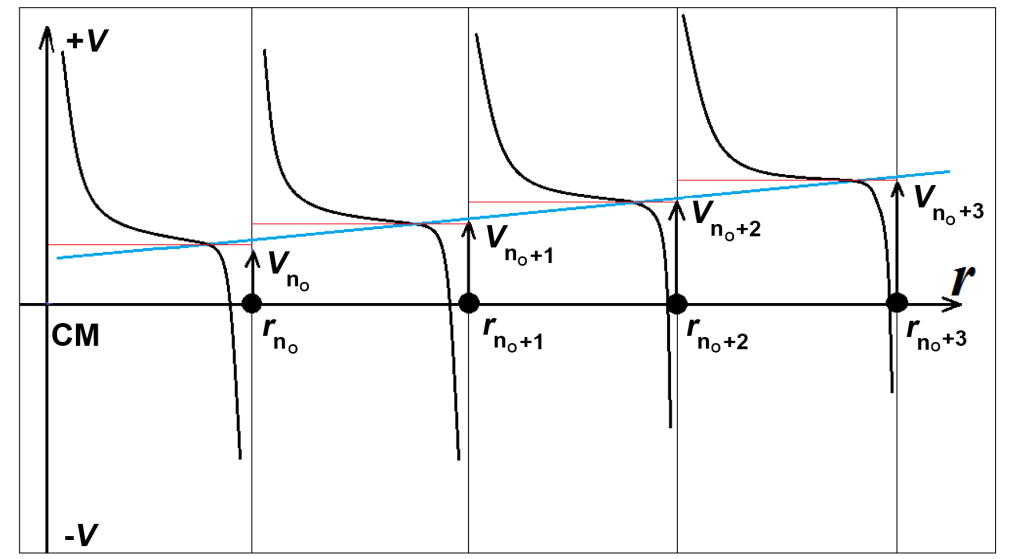

Figure 5. Sketch of the velocity profile of the collective velocity field of the HQS of a system of a large number of concentric star loops as a function of the distance from the galactic center. The sketch shows the velocity profile along one given $r$ coordinate within the loop disk, through four intermediate star loops, for a case, in which the star density is constant with $r$. The distance from the CM to the numbered and equally spaced loops is indicated by $r_{n}(n=1,2,3, \cdots)$. The outward distance on from any given star loop $r_{n}$ is $r-r_{n}$, and the separation between successive concentric loops $r_{n+1}-r_{n}$ may be constant.

important and incredible details. For instance, continuing the blue line in Figure 5 to the left for nearly two loop intervals, the rotation velocity may fall to zero. Continuing to the left, the rotation velocity of the star loops may invert and become retrograde, which strongly enhances the retrograde rotation within the 
central region. In many galaxies such retrograde rotation is observed.

In the star loop model, each star loop creates a velocity step $\Delta V$ in the form of Equation (13), where however $M$, the effective mass depends on the local mass density in the loop. Each star loop contributes to the collective velocity field of the HQS. This contribution however depends on the orbital velocity of the star loop. Within the galactic disk, each star loop acts in the sense of opposing the Keplerian decrease $(1 / r)^{1 / 2}$ of the velocity field. It enhances the prograde velocity field outside each star loop, however depresses the opposite velocity field toward the inside. However, if the orbital velocity inverts, the enhancement inverts too, which stabilizes an internal region with retrograde rotation.

In terms of the gravitational potential, the successive star loops act each one in the sense of lowering the gradient of the collective (negative) gravitational potential. Depending on the mass density, the gravitational potential can become leveled or even fall with $r$, as is generally observed in an internal region and can also take place in some region of $r$ within the galactic disk. From the viewpoint of the current gravitational theories, the stars in the galactic disk too attenuate the slope of the gravitational potential within the galactic disk. However, in these theories the attenuation is much too small to match the observations. The reason is that, in these theories, the effect on the profile of the gravitational potential within the galaxy depends only on the position of the gravitational sources (stars) and not on their orbital velocity.

In the region of $r$, where the star density is constant as a function of $r$, the velocity of the HQS in the collective galactic velocity field as well as the orbital velocity of the stars within the galactic disk necessarily increases with $r$. However, in the real galaxies the mass density variations with $r$ are specific for each galaxy. Depending on the mass density as a function of $r$, an internal region about the galactic center may form that wholly rotates in the retrograde sense. NGC 7331 [16] is a well-known example in which the bulge rotates in a sense opposite to that of the external galactic disk, indicating that the mass density in the disk is especially high. Many other examples exist, in which retrograde rotation in an internal region is observed. The authors of Ref. [16] tried to understand the possible origin of this retrograde rotation in terms of catastrophic collisions, successive aggressions etc. However, none of these attempts was successful and the mystery remains unsolved to present date. In the present HQS gravitational mechanism the steep decrease of the orbital velocity profile near to the galactic center as well as the observed retrograde rotation in the internal region of many galaxies are perfectly natural features of the galactic velocity field that has a straightforward explanation. Beyond the galactic border, where only some (planetary) dwarf-galaxies and globular star clusters orbit round the galaxy, the star density in the galactic disk falls steeply and the galactic velocity field tends to recover the Keplerian $(1 / r)^{1 / 2}$ dependence.

The star loop model, developed here, is only a qualitative description. It however can convincingly explain the observed features of the observed galactic 
gravitational dynamics and even predict incredible details, without the need of dark matter. In particular it predicts the profound depression in the star orbital velocity profile. Such depression is present in the velocity profiles of practically all galaxies. It also predicts the possibility of a central region with retrograde rotation, if the mass density is high enough around this central region.

Although the star loop model is only qualitative, it lets clear that the HQS-dynamic gravitational mechanism is the appropriate physics background that naturally accounts for the non-Keplerian rotation of the galaxies without the need of dark matter. It shows that the stars in the galactic disk strongly attenuate the characteristic slope of the Keplerian velocity field Equation (2), leading to the observed non-Keplerian rotation of the galaxies. It even naturally predicts the retrograde rotation, observed at the center of many galaxies.

The planets in the solar system too constitute a disk round the sun, however a very tenuous one with irregular mass distribution and containing less than $1 \%$ of the matter of the solar system. The disk of the planets however is sufficient to attenuate a little bit the slope of the solar Keplerian velocity field and the gradient of its gravitational potential until the border of the solar system, where it regains the Keplerian dependence. This has the consequence that the gravitational acceleration of the solar field increases a little bit on going out beyond the border of the solar system. This may explain the Pioneer anomaly. The Pioneer anomaly is a very small but well-defined (anomalous) increase in the solar gravitational acceleration on the Pioneer 10 and Pioneer 11 spacecrafts, observed as they moved out in two opposite directions beyond the border of the solar system [17] [18]. To now this anomalous acceleration has no explanation.

The null results of the Michelson light experiments searching for light anisotropy due to the orbital and cosmic motion of earth demonstrate that the solar system, despite its orbital velocity of about $230 \mathrm{~km} / \mathrm{sec}$ round the galactic center, is very closely stationary with respect to the local HQS ruling the inertial motion of matter and the propagation of light. However, our solar system obviously cannot be a privileged exception. All the other stars within the galactic disk must equally be closely com-moving with the local HQS in the galactic non-Keplerian velocity field. This shows that the equator of the galactic velocity field, creating the galactic gravitational field, coincides with the galactic disk. The stars are of course not constrained to move along these circular orbits by gravitational forces. These circular motions also cannot be explained in terms of spacetime curvature of GR. In the view of the HQS dynamics gravitational mechanism, analogously as the planets are carried round the sun by the HQS in the solar Keplerian velocity field, the solar system and the other stars are carried along circular equatorial orbits round the galactic center by the HQS in the galactic velocity field. These stars are locally stationary with respect to the local moving HQS, analogously as earth and the other planets of the solar system are stationary with respect to the local HQS in the solar Keplerian velocity field. This explains the null results of the light anisotropy experiments, searching for light anisotropy with respect to the planet earth in spite of its orbital motion round 
the sun and in spite of the orbital motion of the solar system round the center of the Milky Way galaxy.

Likewise the planets are stationary with respect to the local HQS and carried round the sun by the solar Keplerian velocity field, the stars are stationary and carried round the galactic center by the galactic non-Keplerian velocity field. This explains why the orbital velocity of the planets falls according to $(G M / r)^{1 / 2}$, while the stars in the galactic disk does not fall so. However, both the planets as well as the stars in the galactic disk move along circular orbits without the need of a central force field because it is the HQS itself that so moves. This state of affairs is corroborated by the absence of the gravitational slowing of the GPS clocks by the solar field as well as by the absence of the light anisotropy with respect to the planet earth. However, on the other hand, the observed null results of the light anisotropy experiments on earth show that the solar system, as well as the other stars of the Milky-Way galaxy, is stationary with respect to the local moving HQS in the galactic velocity field. These observations are corroborated too by a large number of other observations, described in Refs. [11] [12] [13] [14] [15] All these observations confirm the signature of the true physical mechanism of gravity in action.

In conclusion, clocks moving with earth and with the solar system or with any other star in the galactic disk are very closely stationary with respect to the local HQS and run all naturally synchronous. They all show very closely the same universal proper time. However, the relative velocities between the planets, stationary with respect to the local HQS in their respective orbits, introduce well known frequency shifts. These frequency shifts however are not due to relative velocity. They are due to wavelength stretching or contraction, due to the deformation of the HQS in the solar Keplerian velocity field along their optical path. It can be shown that these frequency shifts are totally equivalent to the usual Doppler effects for the respective observed relative velocities in the ordinary space.

From a more general viewpoint, the observed isotropy of light, with respect to our planet earth, moving with the solar system in the Milky-Way galaxy and with the Milky-Way galaxy in the local region of space, also demonstrates that the recession between the galaxies and the expansion of the universe necessarily is concomitant with the expansion of the HQS itself. All the astronomical bodies throughout the universe are very nearly stationary with respect to the local moving HQS in the respective gravitational fields as well as with respect to the concomitantly expansion of the universe. This leads to the universality of the laws of physics independently from Einstein's Principle of Relativity. This problem will be the subject of another article on dark energy.

\section{The Desperate Hunt for Dark Matter}

Actually many groups around the world are searching for observational evidence of dark matter in the motion and collision of galaxies and in the motion within 
galactic clusters. Dark matter by definition is not observable via the electromagnetic spectrum. It however is believed to interact with matter via gravity and the weak nuclear force. It thus may indirectly be observable via gravitational perturbations on matter and on light. The gravitational effects of dark matter are believed to be responsible for the non-Keplerian rotation of the galaxies. People also are associating observed anomalous star streams and motions of galaxies with effects by dark matter. Dark matter also is believed to gravitationally affect the propagation of light, causing anomalous gravitational light lensing effects by galaxies and galactic clusters.

All these observations however are much too tenuous to give significant evidence for the existence of the enormous amount of dark matter that is estimated to be five times larger than the whole visible matter-energy in the universe. Some cosmologists [19] [20] suggest that dark matter simply may not exist and that it is a strange need, created by the current flawed theories of gravitation. It also is suggested that gravity is not a fundamental force; however an emergent phenomenon, due to some ignored physical mechanism, like entropic effects.

In the present work, gravity too is not seen as a fundamental force. It is seen as the result of inertial motions (wave propagation) within a moving spatial medium (HQS) that materializes the local Lorentz frames and governs the inertial motion of matter-energy. Likewise the Meissner effect in superconductors, the Higgs mechanism too may develop macroscopic screening velocity fields (currents) of the HQS round the astronomical bodies in the form of Keplerian velocity fields Equation (2) or the non-Keplerian galactic velocity fields, thereby macroscopically confining (expelling out) the matter fields and thereby reducing its energy. The preceding sections show that the velocity field of the HQS, created by the orbiting stars in the galaxies, has a non-Keplerian form. This non-Keplerian velocity field of the HQS straightforwardly explains the non-Keplerian rotation of the galaxies, without the need of dark matter. From this viewpoint, the anomalous star streams, observed near to galaxies and within galactic clusters as well as the apparently anomalous motions of galaxies, are caused simply by the extended velocity fields of the HQS beyond the galactic borders, where this velocity field tends to recover the Keplerian dependence. The dark matter hunters may simply be seeing effects of the HQS tempests, caused by neighboring interacting galaxies and galactic clusters.

\section{Concluding Comments}

The HQS dynamics gravitational mechanism is naturally appropriate to account for the gravitational dynamics in the solar system, without the need of postulating arcane spacetime geometry or postulating exotic dark matter. The non-Keplerian rotation of the galaxies is by far the clearest and most critical observation that the current theories cannot explain. The HQS dynamics gravitational mechanism naturally predicts this non-Keplerian rotation without 
the need of dark matter. No other theory of gravitation can do so, without requiring an enormous amount of exotic dark matter. The present work shows that the velocity of the HQS, within the galactic disk of most galaxies, is nearly constant as a function of the distance $r$ from the galactic center. The velocity gradient of the HQS (central force field) is very low. However, despite the absence of a central force field, the stars move along circular orbits. Within the galactic velocity field of the HQS, the effective velocity $c^{\prime}$ of light is given by $\boldsymbol{c}^{\prime}=\boldsymbol{c}+\boldsymbol{V}$, where $\boldsymbol{c}$ is the fixed velocity of light with respect to the local moving HQS and $\boldsymbol{V}$ is the local velocity of the HQS itself. The effective velocity of light traversing a galactic disk perpendicularly to the disk is expected to be lower. If the direction is not normal to the galactic disk, it also must be additionally delayed in the retrograde side and advanced at the prograde side. It however will undergo no significant change of direction. Hence, the light lensing effect on light traversing the galactic disk, if it exists at all, is very low. However, beyond the border of the galactic disk, the velocity field tends to recover the Keplerian $(1 / r)^{1 / 2}$ dependence. The refraction rate of light, coming through this region from the back of galaxies, may be strong enough to be observed. This effect is analogous to that giving rise to the light lensing effect by the solar field, please see Section 5.5 of Ref. [11]. In fact, the refraction rate outside the galactic border is much smaller than that near to the sun. However, while the light lensing effect by the solar field is produced in about 13.5 milliseconds, in galaxies the excess time delay of light through the retrograde side or time gain in the prograde side may be of days or even of years. It hence can result in considerably large angular deflections. The solar Keplerian velocity field of the HQS along $\phi$ has a large velocity gradient (toward the sun) in the whole region. However, the galactic velocity field has a considerable velocity gradient only beyond the border of the galaxies and of colliding galaxies. Therefore, while the refraction of light by the solar field is relevant through the whole retrograde and also the whole prograde hemispheres, refraction by a galaxy or galactic clusters occurs only in limited regions around the border of the galactic disk. Refraction by the galactic field is typically a non-local effect, because it takes place well outside the gravitational source. This precisely is what is seen in the obtained measurements of the light lensing in galaxies, in colliding galaxies and galactic clusters [21]. The refracted light is diffuse because the galaxies, emitting the light, are extended objects and their lensing cannot produce a well resolved image. An idea that is often seen in the literature, asserts that a galaxy can move through another galaxy with very little interaction, likewise a swarm of birds flying through another swarm of birds. From the view of the present work, this is well far from reality. In the present view, besides possible collisions between stars, there is the collision between the two HQS velocity fields Equation (2). These velocity fields do not obey the principle of superposition, like electromagnetic pulses, however, they add up according to $\left(\boldsymbol{V}_{1}+\boldsymbol{V}_{2}\right)^{1 / 2}$. Therefore, colliding galaxies is a much more complex affair. 


\section{Conflicts of Interest}

The author declares no conflicts of interest regarding the publication of this paper.

\section{References}

[1] Rubin, V. and Ford Jr, W.K. (1970) Astrophysical Journal, 159, 379. https://doi.org/10.1086/150317

[2] Rubin, V., Thonnard, N. and Ford Jr, W.K. (1980) Astrophysical Journal, 238, 471-487. https://doi.org/10.1086/158003

[3] Remmen, G. (2010) Journal of Undergraduate Research in Physics, 19, 1.

[4] Schaf, J. (2017) World Journal of Research and Review (WJRR), 4, 68.

[5] Schaf, J. (2015) Universal Journal of Physics and Application, 9, 189.

[6] Barbanis, B. and Prendergast, K.H. (1966) The Astronomical Journal, 72, 215. https://doi.org/10.1086/110220

[7] Higgs, P.W. (1964) Physical Review Letters, 13, 508. https://doi.org/10.1103/PhysRevLett.13.508

[8] Englert, F. and Brout, R. (1964) Physical Review Letters, 13, 321. https://doi.org/10.1103/PhysRevLett.13.321

[9] Meissner, W. and Ochsenfeld, R. (1933) Naturwissenschaften, 21, 787-788. https://doi.org/10.1007/BF01504252

[10] Anderson, P.W. (1963) Physical Review, 130, 439. https://doi.org/10.1103/PhysRev.130.439

[11] Schaf, J. (2018) Journal of Modern Physics, 9, 1111-1143. https://doi.org/10.4236/jmp.2018.95068

[12] Schaf, J. (2018) Journal of Modern Physics, 9, 395-418. https://doi.org/10.4236/jmp.2018.93028

[13] Schaf, J. (2017) The True Origin of the Gravitational Dynamics. Sc. Res. Publishing, Inc., USA.

[14] Schaf, J. (2015) Universal Journal of Physics and Application, 9, 141.

[15] Schaf, J. (2014) Recent Progress in Space Technology, 4, 44.

[16] Prada, F., Gutierrez, C., Peletier, R.F. and McKeith, C.D. (1996) Astrophysics. arXiv:astro-ph/9602142.

[17] Turyshev, S.G. and Toth, V.T. (2010) General Relativity and Quantum Cosmology. arXiv:1001.3686v1.

[18] Anderson, J.D., Laing, P.A., Lau, E.L., Liu, A.S., Nieto, M.M. and Turyshev, S.G. (2002) Physical Review D, 65, Article ID: 082004. https://doi.org/10.1103/PhysRevD.65.082004

[19] Verlinde, E. (2016) High Energy Physics-Theory; General Relativity and Quantum Cosmology. arXiv:1611.02269v28.

[20] Verlinde, E. (2010) High Energy Physic-Theory. arXiv:1001.0785v1.

[21] Siegel, E. (2017) The Bullet Cluster Proves Dark Matter Exists, But Not for the Reason Most Physicists Think. 